\title{
Serodiagnosis of Chronic Chagas Infection by Using EIE- Recombinant-Chagas-Biomanguinhos Kit
}

\author{
Yara M Gomes $/{ }^{+}$, Valéria RA Pereira, Mineo Nakazawa, Daniela S Rosa, \\ Maria das Neves DS Barros*, Antonio GP Ferreira**, Edimilson D Silva**, \\ Sueli F Yamada Ogatta***, Marco Aurélio Krieger****, \\ Samuel Goldenberg****
}

\begin{abstract}
Departamento de Imunologia, Centro de Pesquisas Aggeu Magalhães-Fiocruz, Av. Moraes Rego s/nº , Cidade Universitária, 50670-420 Recife, PE, Brasil *Ambulatório de Doença de Chagas, Hospital Universitário Oswaldo Cruz-UPE, Recife, PE, Brasil **Laboratório de Reativos do Instituto de Tecnologia em Imunobiológicos, BioManguinhos-Fiocruz, Rio de Janeiro, RJ, Brasil ***Departamento de Microbiologia, Universidade Estadual de Londrina, Londrina, PR, Brasil ****Departamento de Bioquímica e Biologia Molecular, Instituto Oswaldo CruzFiocruz, Rio de Janeiro, RJ, Brasil
\end{abstract}

A kit based on an enzyme immunoassay, EIE-Recombinant-Chagas-Biomanguinhos, developed by the Oswaldo Cruz Foundation, was evaluated for the serodiagnosis of chronic Chagas disease. Evaluation was performed with 368 serum samples collected from individuals living in an endemic area for Chagas disease: 131 patients in the chronic phase with confirmed clinical, epidemiological, and serological diagnosis (indirect immunofluorescence, indirect hemagglutination or enzyme-linked immunosorbent assay) and 237 nonchagasic seronegative individuals were considered negative control. The EIE-Recombinant-Chagas-Biomanguinhos kit showed high sensitivity, 100\% (CI 95\%: 96.4100\%) and high specificity, 100\% (CI 95\%: 98-100\%). The data obtained were in full agreement with clinical and conventional serology data. In addition, no cross-reaction was observed with sera from patients with cutaneous $(n=14)$ and visceral $(n=3)$ leishmaniasis. However, when these sera were tested by conventional serological assays for Chagas disease, cross-reactions were detected in $14.3 \%$ and $33.3 \%$ of the patients with cutaneous and visceral leishmaniasis, respectively. No cross-reactions were observed when sera from nonchagasic seronegative patients bearing other infectious disease (syphilis, $n=8$; $H T L V, n=8 ; H C V, n=7$ and $H B V, n=12$ ) were tested. In addition, sera of patients with inconclusive results for Chagas disease by conventional serology showed results in agreement with clinical evaluation, when tested by the kit. These results are relevant and indicate that the refered kit provides a safe immunodiagnosis of Chagas disease and could be used in blood bank screening.

Key words: serodiagnosis - recombinant antigens - Trypanosoma cruzi - Chagas disease

Chagas disease is still a major health problem in Latin America where 16-18 million individuals are infected with the causative agent Trypanosoma cruzi and at least 90 million people are estimated to be at risk of infection (WHO 1996). Under natural conditions, infected reduviid bugs transmit the T. cruzi to humans when broken skin or mucous membranes contact metacyclics trypomastigotes from insect excreta. However, T. cruzi may bypass the vector bugs and be transmitted to man by a number of alternative mechanisms: blood trans-

This work was partially supported by a PADCT-Finep to MAK. SG is recipient of a research fellowship from CNPq.

${ }^{+}$Corresponding author. Fax: +55-81-453.2449. E-mail: yara@cpqam.fiocruz.br

Received 16 June 2000

Accepted 20 December 2000 fusion, congenital transmission, accidental laboratory contamination, organ transplantation from infected donors and transmission by oral route (Umezawa et al. 1996, Gomes 1997). Blood transfusion is the second most common means of infection and the human migration from endemic areas to urban centers is proving a rising risk of transfusional Chagas disease in all Latin America and in non endemic countries (Schmuñis 1991).

Diagnosis of chronic Chagas disease is based on the detection of parasite by indirect parasitological methods (xenodiagnosis and hemoculture) or more usually on the detection of IgG antibodies against $T$. cruzi in the sera of patients by immunological methods (complement fixation-CF, indirect immunofluorescence-IIF, direct agglutination-DA, indirect hemagglutination-IHA and enzyme-linked immunosorbent assay-ELISA). The methods based on detection of the parasite, although highly specific, are of limited sensitivity, because parasites are 
detected in only $20-50 \%$ of individuals known to be infected, resulting in many false negative results (Gomes 1997). On the other hand, the methods based on the detection of an immune response to the parasite in the mamalian host lack specificity since they use crude or partially purified parasite extracts. Cross-reaction to $T$. cruzi has been observed with related protozoan diseases, particularly leishmaniasis. The problems with conventional assays $(\mathrm{CF}$, IHA, DA, IIF and ELISA) may be overcome by using recombinant polypeptides containing specific T. cruzi epitopes that elicit an immune response in the majority of chagasic patients.

Several T. cruzi genes have been cloned and some of recombinant antigens have been assayed for their use in diagnosis (Affranchino et al. 1989, Levin et al. 1989, Almeida et al. 1990, Paranhos et al. 1990, Cotrin et al. 1990, Zingales et al. 1990, Goldenberg et al. 1991, Umezawa et al. 1999). Two recombinant antigens, CRA and FRA, expressed in the bacterium Escherichia coli were analyzed by Krieger et al. (1992) in a diagnostic test for Chagas disease. The data indicated that recombinant antigens displayed better results when used in combination than separately. These authors developed a direct ELISA which involves the use of peroxidase-labeled antigens to detect the immunecomplexes. The results indicate that the recombinant (CRA+FRA) ELISA was better than the conventional ELISA in the diagnosis of Chagas disease, providing $100 \%$ specificity and sensitivity in all sera tested (Almeida et al. 1990, Krieger et al. 1992). These antigens were characterized and shown to display a repetitive epitope structure (Lafaille et al. 1989, Krieger et al. 1990). FRA (flagellar repetitive antigen) is located in the flagellum of the parasite and displays a 68-amino acid repeat, while CRA (cytoplasmic repetitive antigen) is distributed throughout the cytoplasm and has a 14-amino acid repeat (Lafaille et al. 1989).

Recently, a kit for diagnosis of chronic Chagas disease, using CRA+FRA antigens was developed by Oswaldo Cruz Foundation (Fiocruz), Rio de Janeiro, Brazil. The kit is based on enzyme immunoassay, the direct ELISA.

In the present work we report the evaluation of the EIE-Recombinant-Chagas-Biomanguinhos kit for the diagnosis of $T$. cruzi infection using characterized serum samples from individuals living in Chagas disease endemic areas and from individuals with other infectious diseases.

\section{MATERIALS AND METHODS}

Human sera - Serum samples were collected from 368 patients between the ages of 5 and 76 years old from Hospital Universitário Oswaldo Cruz (Recife) living in Chagas disease endemic areas in the State of Pernambuco, Brazil: 131 pa- tients in the chronic phase of the Chagas disease with confirmed clinical, epidemiological, and serological diagnosis and 237 nonchagasic individuals with negative serology were considered negative controls. Serum samples were previously classified as negative when two serological tests (ELISA, IIF or HAI) gave nonreactive results against $T$. cruzi antigens and as positive when two tests were reactive. Serum samples of 14 patients with cutaneous leishmaniasis (CL) and 3 with visceral leishmaniasis (VL) were also tested. Diagnosis of CL was based upon the collective analysis of a set of elements: presence of typical lesions, compatible epidemiological history and direct parasite detection. VL was diagnosed by clinical, epidemiological history, positive serological assay (IIF) and by detection of parasites in bone marrow aspirate. These sera were also tested by conventional serological assay to Chagas disease (ELISA and HAI) to evaluate cross-reaction.

Sera from patients with other infectious disease (8 with syphilis, 8 with HTLV, 7 with HCV, and 12 with HBV) as well as sera of patients with inconclusive results for Chagas disease (Table II) from blood center Fundação Hemope/Hemocentro, Pernambuco, Brazil were included in this study. All sera were analyzed by EIE-RecombinantChagas-Biomanguinhos kit. Blood samples from the individuals were taken by venopuncture and the sera obtained were stored at $-20^{\circ} \mathrm{C}$ until use.

Enzyme immunoassay - The enzyme immunoassay, with the EIE-Recombinant-ChagasBiomanguinhos, was performed according to manufacturer. Briefly, microplates sensitized with the recombinant antigens were incubated with undiluted patient sera $(50 \mu \mathrm{l})$ at $37^{\circ} \mathrm{C}$ for $30 \mathrm{~min}$. After washes to remove the unbound antibodies, the plates were incubated for $30 \mathrm{~min}$ at $37^{\circ} \mathrm{C}$ with 50 $\mu \mathrm{l}$ of peroxidase conjugated antigens. After repeated cycles of washes the immune complexes were revealed by the addition of hydrogen peroxide and 3, 3', 5, 5' - tetramethylbenzidine. The reaction was stopped with $2 \mathrm{M} \mathrm{H}_{2} \mathrm{SO}_{4}$, and the optical density (OD) at $450 \mathrm{~nm}$ was determined in a ELISA reader (Bio-Rad 3550). The cutoff (CO) values as well as the gray zone were calculated for each plate according to manufacturer. Sera with $\mathrm{OD}$ values equal or greater than $\mathrm{CO}$ value were considered reactive, and consequently considered positive for antibodies to $T$. cruzi. Sera with OD values below $\mathrm{CO}$ were considered non-reactive and negative for antibodies to $T$. cruzi.

Data analysis - The figure of samples recorded as $\mathrm{OD}_{450 \mathrm{~nm}}$ was distributed by using computer graphics software. The values of sensitivity and specificity were calculated according to Camargo (1992). The confidence interval (CI) was calculated at the level of $95 \%$. 


\section{RESULTS}

ELISA results are shown in the Figure. The CO and the gray zone values for each plate are shown in Table I. The high sensitivity of $100 \%$ (CI 95\%: 96.4-100\%) and specificity of 100\% (CI 95\%: 98$100 \%$ ) show the excellent performance of the EIERecombinant-Chagas-Biomanguinhos kit. All 131 cases of confirmed Chagas disease, which had been diagnosed by clinical and conventional serology were positive and all 237 sera from nonchagasic individuals were negative.

Analysis of the sera negative for Chagas disease but positive for CL and VL shows that the responses do not give rise to false positive results (Figure). When these sera were tested by conventional serological assays for Chagas disease, crossreactions were observed in $14.3 \%(2 / 14)$ of the patients with CL and $33.3 \%(1 / 3)$ patients with VL. No cross-reactions were observed when sera from patients with syphilis, HTLV, HBV and HCV were tested by the recombinant kit. The sera with inconclusive results for Chagas disease by using conventional serological assays were in agreement with the clinical evaluation (two chagasic patients and four nonchagasic individuals) when tested by recombinant kit (Table II).

\section{TABLE I}

Cutoff and gray zone values obtained for each plate in present report

\begin{tabular}{lcc}
\hline Plates & $\begin{array}{c}\text { Cutoff values } \\
(\mathrm{OD})\end{array}$ & $\begin{array}{c}\text { Gray zone values } \\
(\mathrm{OD})\end{array}$ \\
\hline 1 & 0.233 & $0.233-0.279$ \\
2 & 0.244 & $0.244-0.293$ \\
3 & 0.198 & $0.198-0.237$ \\
4 & 0.192 & $0.192-0.231$ \\
5 & 0.178 & $0.178-0.213$ \\
6 & 0.183 & $0.183-0.220$ \\
\hline
\end{tabular}

\section{TABLE II}

Comparative evaluation of the sera with the inconclusive results by conventional serological tests and EIE-Recombinant-Chagas-Biomanguinhos kit

\begin{tabular}{lccc}
\hline Sera & \multicolumn{2}{c}{$\begin{array}{c}\text { Conventional } \\
\text { serological tests }\end{array}$} & \multirow{2}{*}{ EIE-Recombinant-kit } \\
\cline { 2 - 3 } & $\begin{array}{c}\text { ELISA } \\
\text { OD/CO }\end{array}$ & IIF & \\
& & OD/CO \\
\hline $1^{a}$ & $0.289 / 0.180$ & $\mathrm{NR}$ & $0.087 / 0.198$ \\
$2^{a}$ & $0.245 / 0.180$ & $\mathrm{NR}$ & $0.087 / 0.198$ \\
$3^{a}$ & $0.286 / 0.180$ & $\mathrm{NR}$ & $0.088 / 0.198$ \\
$4^{a}$ & $0.254 / 0.180$ & $\mathrm{NR}$ & $0.096 / 0.198$ \\
$6^{b}$ & $1.188 / 0.180$ & $\mathrm{NR}$ & $1.242 / 0.198$ \\
$7^{b}$ & $0.215 / 0.180$ & $\mathrm{R}$ & $1.150 / 0.198$ \\
\hline
\end{tabular}

$a$ : nonchagasic sera; $b$ : chagasic sera; NR: non reactive sera; R: reactive sera; OD: optical densities; CO: cutoff.

\section{DISCUSSION}

The serological conventional assays (CF, IIF, IHA and ELISA) for diagnosis of Chagas disease are used for individual diagnosis and for screening of donated blood, as well as in epidemiological studies. However, a persistent problem with these conventional assays has been the occurrence of false-positive results. Due to this problem, the World Health Organization recommends that serum specimens should be tested in two conventional assays before being accepted as positive (Gomes 1997). This approach carries with it an enormous logistic and economic burden, especially for a blood bank. Even if some blood banks use three different serological tests to reach a diagnosis, the amount of blood discarded that could be transfused, if there was a safe test, is significant. As an example, in the major Brazilian blood center (Fundação Pró-Sangue Hemocentro de São Paulo) some 10,000 blood units are discarded per year due their reactivity for Chagas disease in conventional serologic tests, at a cost of approximately U\$ 60.00 per bag (Carvalho et al. 1993). In addition, the diagnostic tests that give some false-positive results should be avoided because such tests can create social problems for false-positive chagasic patients. They can also produce erroneous data in epidemiological studies (Krieger et al. 1992).

In order to overcome these problems, a new diagnostic kit EIE-Recombinant-ChagasBiomanguinhos was developed by Fiocruz to detect antibody to $T$. cruzi in sera and plasma. The test is a direct ELISA that use the CRA+FRA recombinant antigens.

In the present report we have evaluated the EIERecombinant-Chagas-Biomanguinhos kit using serum samples from individuals living in Chagas disease endemic areas of State of Pernambuco, Brazil. The test showed to be highly sensitive and specific, detecting $100 \%$ of the chagasic (131/131) and nonchagasic individuals from endemic areas (237/237), respectively. Sensitivity and specificity were $100 \%$ (CI 95\%: 96.4-100\%) and 100\% (CI 95\%: 98-100\%), respectively. In addition, no cross-reaction was observed with sera from patients with CL and VL. Even if low reactivity sera were not included in this study, we may predict a high sensitivity according to the results obtained with these confirmed chagasic patients. Several conventional serological tests as well as ELISAs commercially available in Brazil showed cross-reactivity to sera from patients with other diseases. These false-positive results are frequent in the case of patients with leishmaniasis (Carvalho et al. 1993). The absence of cross-reactions in EIE-Recombi- 
nant-Chagas-Biomanguinhos shows its high specificity for Chagas disease.

According to Camargo (1992), a problem that occurs during screening of chagasic sera in blood banks is the variable percentage of samples showing a reactivity in the gray zone around the cutoff values. In order to verify this problem we tested by recombinant kit, sera from blood center Fundação Hemope/Hemocentro, Pernambuco, Brazil with inconclusive results for Chagas disease obtained by conventional serological assays. Reactivity in the gray zone was not observed and the results were in full agreement with clinical evalu- ation. The kit EIE-Recombinant-ChagasBiomanguinhos for the diagnosis of chronic Chagas disease has several advantages over other available methods: (i) the use of specific $T$. cruzi recombinant antigens avoids false-positive reaction; (ii) the direct ELISA increases the sensitivity of the method allowing the evaluation of low titer sera, and corroborates its specificity; (iii) the use of undiluted serum samples reduces the possibility of error due to manipulation; (iv) the procedure is quick (taking $2 \mathrm{~h}$ to perform), easily performed and reliable. Taken as a whole, these facts indicate that the EIE-Recombinant-Chagas-Biomanguinhos is

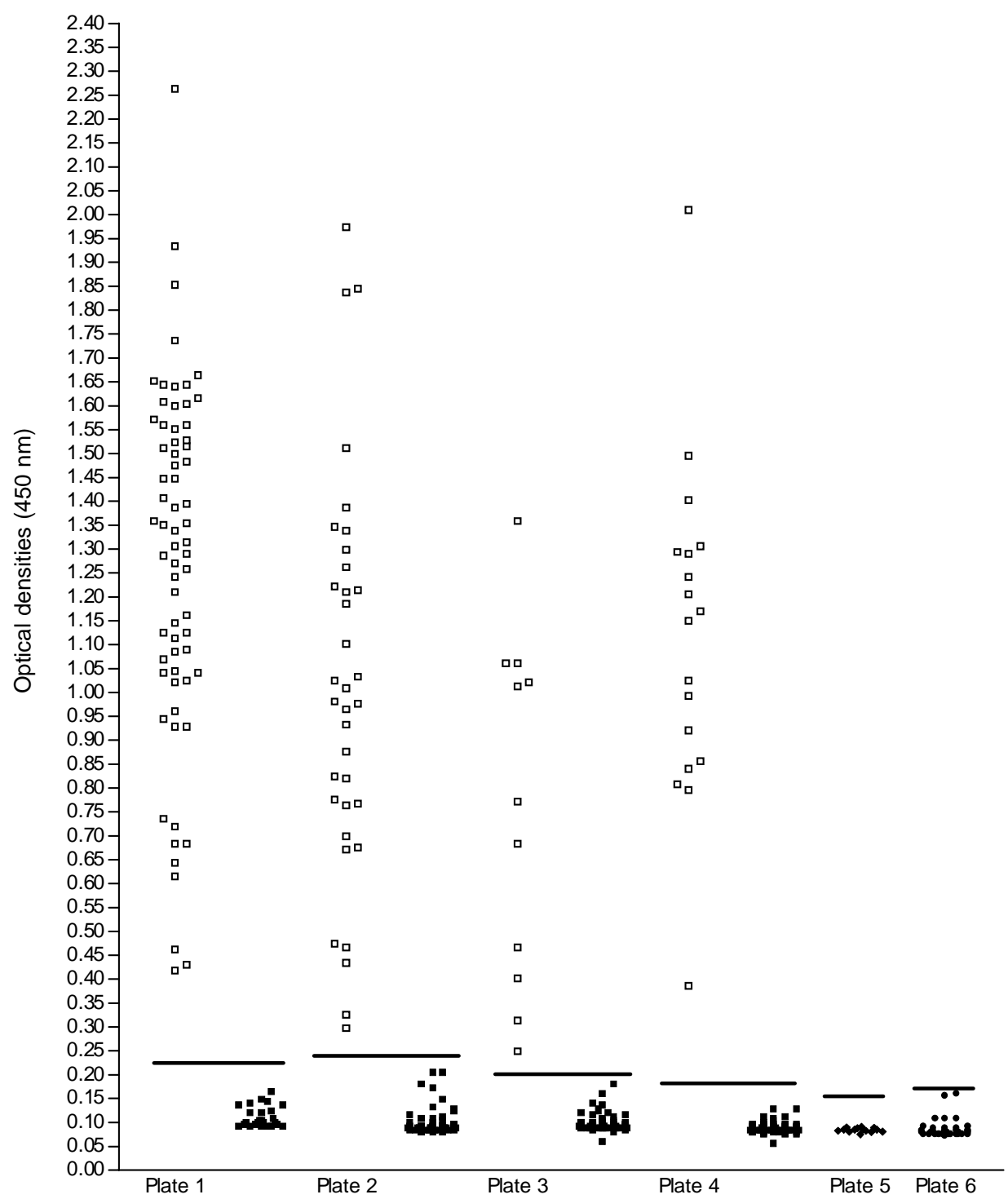

Distribution of optical densities values from chagasic and nonchagasic individuals using the EIE-Recombinant-ChagasBiomanguinhos kit. The horizontal line inside the drops for each plate represents the cutoff values. Chagasic individuals ( $\square$ ); nonchagasic individuals ( $\square$ ); individuals with visceral and cutaneous leishmaniasis ( )); syphilis, HTLV, HCV and HBV infections $(\bullet)$ 
suitable for the diagnosis of Chagas disease and could be used in blood bank screening. We are currently evaluating this kit for screening donated blood of the Fundação Hemope.

\section{ACKNOWLEDGEMENTS}

To Edileuza Brito for providing sera of leishmaniasis and Wayner Souza for performing the statistical analysis. To Genilda Medeiros and Ana Cristina B Souza of the blood center Fundação Hemope/Hemocentro, Pernambuco for performing several serological assays for Chagas disease and providing sera with other infectious diseases.

\section{REFERENCES}

Affranchino JL, Ibanez CF, Luquetti AO, Rassi A, Ryes MB, Macina RA, Aslund L, Petterson U, Frasch ACC 1989. Identification of a Trypanosoma cruzi antigen that is shed during the acute phase of Chagas' disease. Mol Biochem Parasitol 34: 221-228.

Almeida E, Krieger MA, Carvalho MR, Oelemann W, Goldenberg S 1990. Use of recombinant antigens for the diagnosis of Chagas' disease and blood bank screening. Mem Inst Oswaldo Cruz 85: 513-517.

Camargo ME 1992. An appraisal of Chagas' disease serodiagnosis. In S Wendel, Z Brener, ME Camargo, A Rassi (eds), Chagas' Disease (American Trypanosomiasis): its Impact on Transfusion and Clinical Medicine, ISBT Brazil'92, São Paulo, p. 165168.

Carvalho MR, Krieger MA, Almeida E, Oelemann W, Shikanai-Yassuda MA, Ferreira AW, Pereira JB, Sáez-Alquézar A, Dorlhiac-Llacer PE, Chamone DF, Goldenberg S 1993. Chagas' disease diagnosis: evaluation of several tests in blood bank screening. Transfusion 33: 830-834.

Cotrim PC, Paranhos GS, Mortara RA, Wanderley J, Rassi A, Camargo ME, Franco da Silveira J 1990. Expression in Escherichia coli of a dominant immunogen of Trypanosoma cruzi recognized by human chagasic sera. J Clin Microbiol 28: 519-524.

Goldenberg S, Krieger MA, Lafaille JJ, Almeida E, Oelemann W 1991. Use of Trypanosoma cruzi antigens in the immunological diagnosis of Chagas' disease. Mem Inst Butantan 53: 71-76.

Gomes YM 1997. PCR and sero-diagnosis in chronic Chagas'disease: biotechnological advances. Appl Biochem Biotechnol 66: 107-119.

Krieger MA, Salles JM, Almeida E, Linss J, Bonaldo
MC, Goldenberg S 1990. Expression and polymorphism of a Trypanosoma cruzi gene encoding a cytoplasmic repetitive antigen. Exp Parasitol 70: 247254.

Krieger MA, Almeida E, Oelemann W, Lafaille JJ, Pereira JB, Carvalho MR, Goldenberg S 1992. Use of recombinant antigens for the accurate immunodiagnosis of Chagas' disease. Am J Trop Med Hyg 46: 427-434.

Lafaille JJ, Linss J, Krieger MA, Souto-Padron T, de Souza W, Goldenberg S 1989. Structure and expression of two Trypanosoma cruzi genes encoding antigenic proteins bearing repetitive epitopes. $\mathrm{Mol}$ Biochem Parasitol 35: 127-136.

Levin M, Mesri E, Benarous R, Levitus G, Schijman A, Leyati P, Chiale P, Ruiz AM, Khan A, Rosenbaum M, Torres HN, Segura EL 1989. Identification of major Trypanosoma cruzi antigenic determinants in chronic Chagas' disease. Am J Trop Med Hyg 41: 530-538.

Paranhos GS, Cotrin PC, Mortara RA, Rassi A, Corral R, Freilij HL, Grinstein S, Wanderley J, Camargo ME, Franco da Silveira J 1990. Trypanosoma cruzi: cloning and expression of an antigen recognized by acute and chronic human chagasic sera. Exp Parasitol 71: 284-293.

Schmuñis GA 1991. Trypanosoma cruzi, the etiologic agent of Chagas' disease: status in the blood supply in endemic and nonendemic countries. Transfusion 31: 547-557.

Umezawa ES, Nascimento MS, Kersper Jr N, Coura JR, Borges-Pereira J, Junqueira ACV, Camargo ME 1996. Immunoblot assay using excreted-secreted antigens of Trypanosoma cruzi in serodiagnosis of congenital, acute and chronic Chagas'disease. J Clin Microbiol 34: 2143-2147.

Umezawa ES, Bastos S, Camargo ME, Yamauchi LM, Santos MR, Gonzalez A, Zingales B, Levin MJ, Sousa O, Rangel-Aldao R, da Silveira JF 1999. Evaluation of recombinant antigens for serodiagnosis of Chagas' disease in South and Central America. J Clin Microbiol 37: 1554-1560.

WHO-World Health Organization 1996. Control of Tropical Disease. Chagas' Disease. A Disease whose Days are Numbered, WHO, Geneve, 16 pp.

Zingales B, Gruber A, Ramalho CB, Umezawa ES, Colli W 1990. Use of recombinant proteins of Trypanosoma cruzi in the serological diagnosis of Chagas' disease. Mem Inst Osvaldo Cruz 85: 519-522. 